\title{
HaMiPla Best Paper Sitzungen - Ihre Favoriten 2015
}

\section{HaMiPla Best Paper Award - Your Favourites in 2015}

K. Maier, R. E. Giunta,

K. J. Prommersberger
Bibliografie

DOI http://dx.doi.org/

10.1055/s-0042-119655

Handchir Mikrochir Plast Chir 2016; 48: 377-378

(c) Georg Thieme Verlag KG Stuttgart · New York

ISSN 0722-1819

Korrespondenzadresse

\section{Katrin Maier}

Programmbereichsleiterin Georg Thieme Verlag KG Medizin

Rüdigerstraße 14 70469 Stuttgart katrin.maier@thieme.de
Mit dem HaMiPla Best-Paper-Award zeichnen wir jedes Jahr die Top Publikationen in Handchirurgie und Plastischer Chirurgie aus, die unsere Leser und Nutzer am meisten interessiert haben. Die Verleihung des Preises erfolgt im Rahmen der wissenschaftlichen Sitzungen auf der DGPRÄC und VDÄPC Jahrestagung und dem DGH Kongress.

Den Auftakt hatte in diesem Jahr die plastisch chirurgische Sitzung unter dem Vorsitz von R. E. Giunta (München), K. J. Prommersberger (Bad Neustadt), M. Haerle (Markgröningen) mit einem sehr abwechslungsreichen Programm am 9. September 2016 in Kassel ( $\bullet$ Abb. 1).

Im ersten Vortrag gab Michael Sauberbier für die Autorengruppe T. Schloßhauer et al. einen sehr anschaulichen und detaillierten Überblick über die Chancen und Herausforderungen beim plastisch-chirurgischen Management von Weichgebesarkomen der unteren Extremität [7].

Einen Bericht aus der Forschung präsentierte Sebastian Dunda aus Berlin und nahm das Auditorium mit in die Welt des 3D Drucks und der Zellkultur. Sein Artikel zur in vitro und in vivo Biokompatibilität einer neuartigen, 3-dimensionalen CelluloseMatrixstruktur war auch bei den Lesern der HaMiPla sehr beliebt [1].

Um auf Patientenfragen künftig fundierter antworten zu können, hat sich die Autorengruppe aus Ludwigshafen mit dem Thema auseinandergesetzt, welche Topischen Externa zur Behandlung von hypertrophen Narben wie gut wirken. Die interessante Übersicht wurde vom Erstautor Sebastian Fischer vorgestellt und unter den Anwesenden munter diskutiert [3].

Den Abschluss bildete der Vortrag von Cornelius Schubert, der die Ergebnisse aus der Münchner Arbeitsgruppe vorstellte, welche Auswirkungen die Eigenständigkeit der universitären plastischen Chirurgie auf das Publikationsverhalten

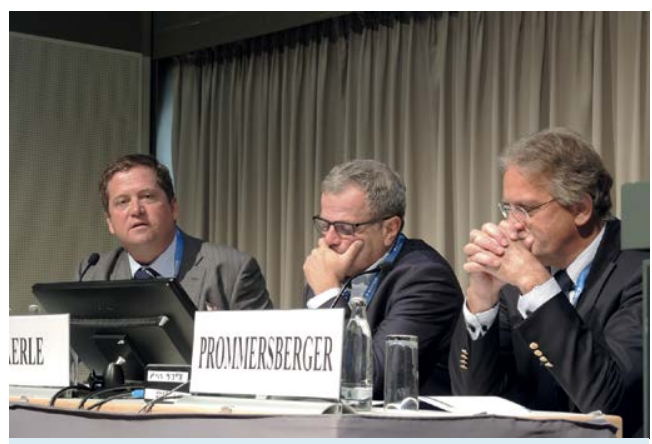

Abb. 1 Die Moderatoren der Sitzung: R. Giunta, M. Haerle und K.-J. Prommersberger bei der „HaMiPla-Best-Paper-Award-2015-Sitzung“ auf dem DGPRÄC Kongress in Kassel 2016 und damit auf die Akquise von Forschungsgeldern hat. Eine spannende Untersuchung, deren Ergebnisse sehr wichtig für das Fach sind [8].

Maximilian Otte als Erstautor des Artikels über DIEP Lappen als Standard zur Brustrekonstruktion war leider verhindert und konnte seine $\mathrm{Ar}$ beit nicht im Rahmen der Sitzung vorstellen [6]. Insgesamt eine spannende und interessante Session, die gezeigt hat, wie viele wichtige Themen in der HaMiPla publiziert und von den Lesern genutzt werden ( $\bullet$ Abb. 2 ).

Der zweite Teil der HaMiPla Best Paper Awards 2015 Sitzung fand in Frankfurt statt im Rahmen des DGH Kongresses am 23. September 2016. Auch hier wurden die 5 Veröffentlichungen, die am häufigsten online genutzt wurden, von den Autoren präsentiert. Der thematische Schwerpunkt lag hierbei auf handchirurgischen Themen. R.E. Giunta und K.J. Prommersberger hatten den Vorsitz und moderierten sehr launig die Diskussionen, die sich über das gesamte Auditorium entspannten.

Die Behandlung der Rhizarthrose mit allen Schwierigkeiten ist - oft mühsamer - handchirurgischer Alltag, und spiegelte sich auch in den präsentierten Arbeiten wider.

Lukas Fatzer stellte die Erfahrungen seiner Autorengruppe vor, die sie mit Distraktionsarthroplastiken nach Bufalini und Perugia bei der Rhizarthrose im Frühstadium machten. Ein Vorgehen mit dem sie gute Ergebnisse erzielen [2]. (॰ Abb. 3-5).

Milena Dettmer berichtete für Annika Zschöck-Holle und die Arbeitsgruppe aus Frankfurt über die Behandlung der Rhizarthrose mittels TrapeziumResektion und Swanson-Silikonprothese. Dieser Vortrag wurde anschließend ebenfalls im Auditorium intensiv diskutiert, da es unter den Anwesenden unterschiedliche Vorgehensweisen gab [10].

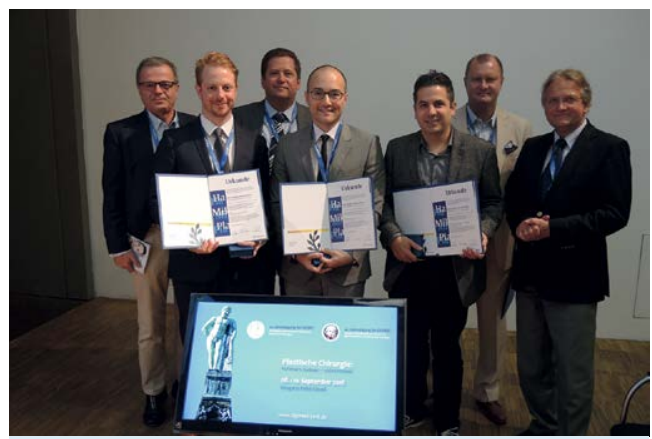

Abb. 2 Preisträger und Referenten der HaMiPla-Best-Paper-Award-Session auf dem DGPRÄC Kongress 2016. Von links nach rechts: M. Haerle, C. Schubert, R. Giunta (Herausgeber HaMiPla),

S. Fischer, S. Dunda, M. Sauerbier, K.-J. Prommersberger (Herausgeber HaMiPla) 


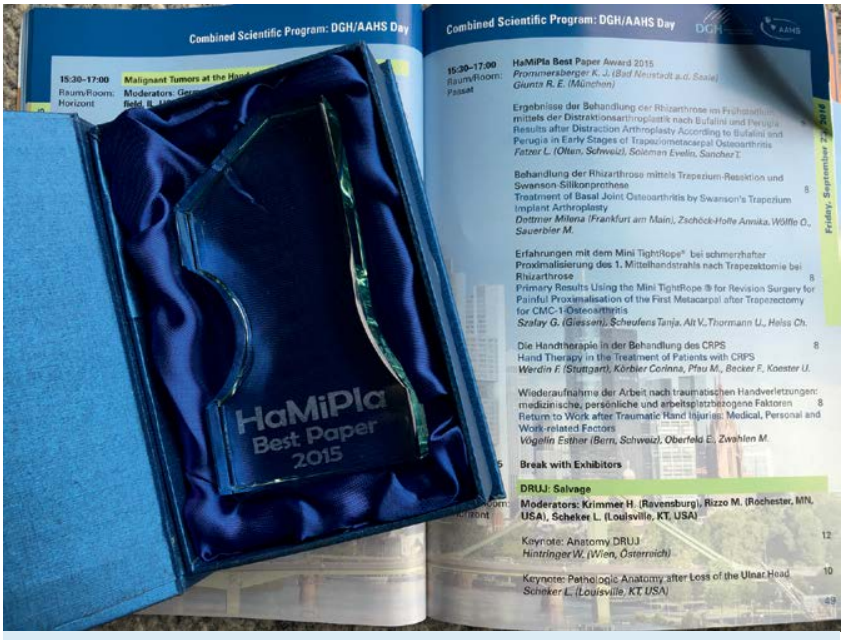

Abb. 3 Ein spannendes Programm auf dem DGH Kongress...

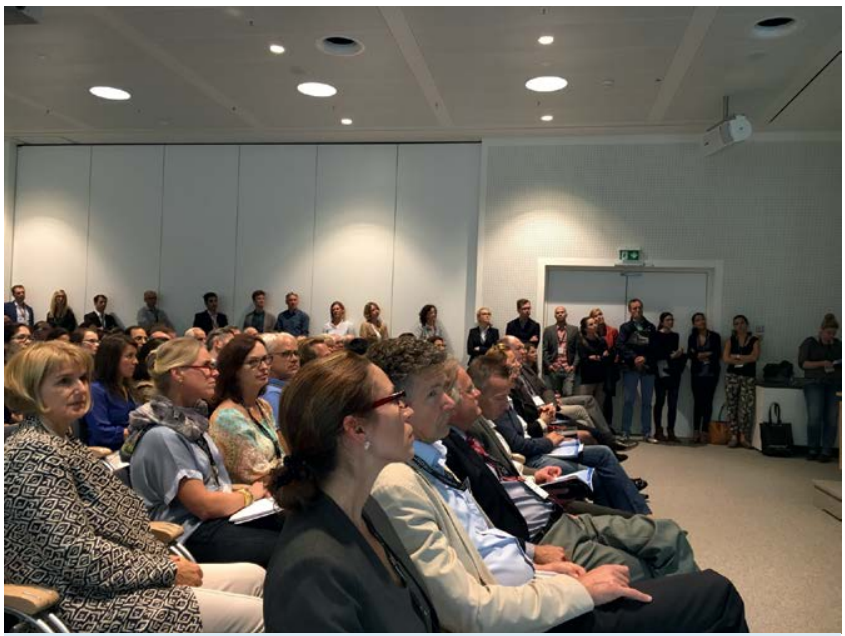

Abb. 4 Ein interessiertes Publikum auf dem DGH Kongress.

Den thematischen Abschluss zur Rhizarthrose machte dann Gabor Szalay mit seinem Vortrag zu den Erfahrungen mit dem Mini TightRope ${ }^{\circledR}$ bei schmerzhafter Proximalisierung des 1. Mittelhandstrahls nach Trapezektomie bei Rhizarthrose [9].

Corinna Körbler stellt die Arbeit von F. Werdin und der Arbeitsgruppe aus Stuttgart vor, die die Aspekte der Handtherapie bei CRPS untersuchten. Auch hier entspann sich eine intensive Diskussion über die unterschiedlichen Erfahrungen und Vorgehensweisen bei der Therapie im Publikum [4].

Esther Vögelin und Kollegen untersuchten die verschiedenen medizinischen, persönlichen und arbeitsplatzbezogenen Faktoren, die eine Rolle spielen für die Wiederaufnahme der Arbeit nach traumatischen Handverletzungen. Eine sehr interessante Übersicht, die den Abschluss der diesjährigen Award-Verleihung bildete [5].

Die 10 Artikel können über folgenden

Link abgerufen werden:

http://tinyurl.com/p3gdlue

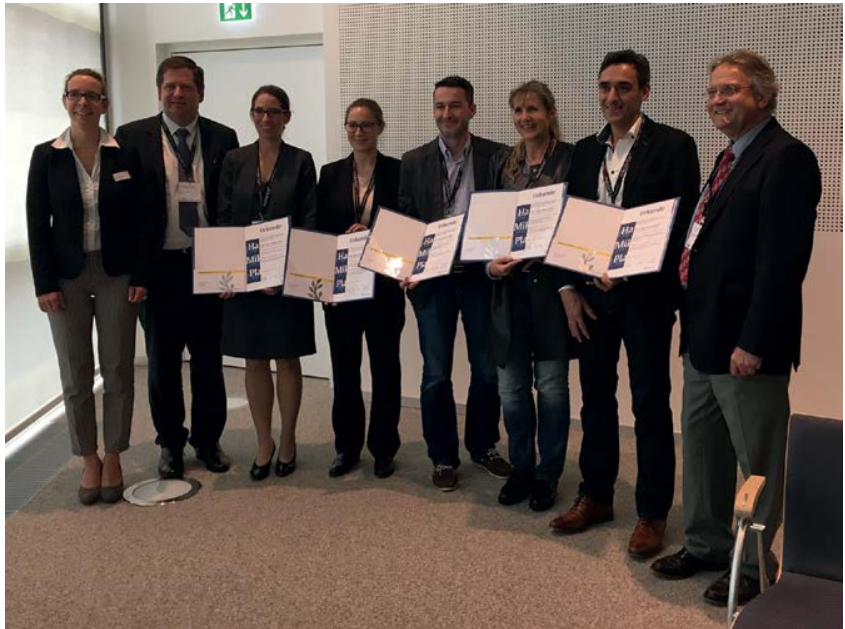

Abb. 5 Preisträger und Referenten der HaMiPla-Best-Paper-Award-Session auf der DGH Jahrestagung 2016. K. Maier (Thieme Verlag), R. Giunta (Herausgeber), C. Körbler, M. Dettmer, L. Fatzer, E. Vögelin, G. Szaray, K.-J. Prommersberger (Herausgeber)

Interessenkonflikt: Nein.

\section{Literatur}

1 Dunda SE, Ranker M, Pallua $N$ et al. In vitro und in vivo Biokompatibilität einer neuartigen, 3-dimensionalen Cellulose-Matrixstruktur. Handchir Mikrochir Plast Chir 2015; 47: 378-383

2 Fatzer L, Soleman E, Sanchez T. Ergebnisse der Behandlung der Rhizarthrose im Frühstadium mittels der Distraktionsarthroplastik nach Bufalini und Perugia. Handchir Mikrochir Plast Chir 2015; 47: 1-6

3 Fischer S, Ernst HR, Drücke D et al. Topische Externa zur Prävention und Therapie hypertropher Narben und Keloide: Eine Literaturrecherche. Handchir Mikrochir Plast Chir 2015; 47: 253-267

4 Körbler C, Pfau M, Becker F et al. Die Handtherapie in der Behandlung des CRPS. Handchir Mikrochir Plast Chir 2015; 47: 182-189

5 Oberfeld E, Zwahlen M, Vögelin E. Wiederaufnahme der Arbeit nach traumatischen Handverletzungen: medizinische, persönliche und arbeitsplatzbezogene Faktoren. Handchir Mikrochir Plast Chir 2015; 47: $44-57$

6 Otte M, Andree C, Hagouan $M$ et al. Der DIEP Lappen als Standard in der autologen Brustrekonstruktion - Ergebnisse und Algorithmus zur erfolgreichen Rekonstruktion. Handchir Mikrochir Plast Chir 2015; 47: $222-227$

7 Schloßhauer T, Mehling IM, Moll $W$ et al. Plastisch-chirurgisches Management bei Weichgewebesarkomen an der unteren Extremität. Handchir Mikrochir Plast Chir 2015; 47: 100-110

8 Schubert CD, Leitsch S, Haertnagl F et al. Vorteil durch Eigenständigkeit? Analyse der Publikationsleistung der universitären Plastischen Chirurgie in verschiedenen Organisationsstrukturen. Handchir Mikrochir Plast Chir 2015; 47: 213-221

9 Szalay G, Scheufens T, Alt $V$ et al. Erfahrungen mit dem Mini TightRope $^{\circledR}$ bei schmerzhafter Proximalisierung des 1 . Mittelhandstrahls nach Trapezektomie bei Rhizarthrose. Handchir Mikrochir Plast Chir 2015; 47: 17-23

10 Zschöck-Holle A, Reik M, Wölfle $O$ et al. Behandlung der Rhizarthrose mittels Trapezium-Resektion und Swanson-Silikonprothese. Handchir Mikrochir Plast Chir 2015; 47: 7-16 